\title{
A precision on the concept of strict convexity in non-Archimedean analysis
}

\author{
Javier Cabello Sánchez, José Navarro Garmendia*
}

\begin{abstract}
We prove that the only non-Archimedean strictly convex spaces are the zero space and the one-dimensional linear space over $\mathbb{Z} / 3 \mathbb{Z}$, with any of its trivial norms.
\end{abstract}

\section{Introduction}

In order to find a non-Archimedean version of the Mazur-Ulam theorem, M. Moslehian and G. Sadeghi introduced in [2] the class of non-Archimedean strictly convex spaces.

Later on, A. Kubzdela observed that the only non-Archimedean strictly convex space over a field with a non-trivial valuation is the zero space ([1, Theorem 2]).

In this note, we prove:

Proposition 2.3 The only non-Archimedean strictly convex spaces are the zero space and the one-dimensional linear space over $\mathbb{Z} / 3 \mathbb{Z}$, with any of its trivial norms.

\section{Non-Archimedean strictly convex spaces}

Firstly, recall that on a non-Archimedean normed space $X$, "any triangle is isosceles"; that is to say, for any $x, y \in X$,

$$
\|y\|<\|x\| \quad \Rightarrow \quad\|x+y\|=\|x\|
$$

Definition $2.1([\mathbf{2}])$. A non-Archimedean normed space $X$ over a field $\mathbb{K}$ is strictly convex if (SC1) $|2|=1$.

(SC2) for any pair of vectors $x, y \in X,\|x\|=\|y\|=\|x+y\|$ implies $x=y$.

Observe that (SC2) may also be rephrased by saying that there are no equilateral triangles; that is to say, that for any pair of distinct vectors $x \neq y \in X$,

$$
\|y\|=\|x\| \quad \Rightarrow \quad\|x+y\|<\|x\| .
$$

Keywords: Mazur-Ulam Theorem; non-Archimedean normed spaces; strict convexity.

Mathematics Subject Classification: 46S10, 26E30. 
EXAMPLE 2.2. If $X$ is a one-dimensional normed linear space over a finite field, then its norm is trivial; i.e., there exists $a \in(0, \infty)$ such that $\|x\|=a$ for every nonzero vector $x \in X$.

The one-dimensional linear space over $\mathbb{Z} / 3 \mathbb{Z}$, with any of its possible trivial norms, is a strictly convex space, in the sense of the Definition above.

Proposition 2.3. If $X$ is a non-zero strictly convex space, then it is linearly isometric to a one-dimensional normed space over $\mathbb{Z} / 3 \mathbb{Z}$.

PROOF. First of all, observe that a strictly convex space can only occur in characteristic 3 : for any vector $x \in X$,

$$
\|2 x\|=\|x\|=\|-x\|
$$

as $2 x+(-x)=x$, condition (SC2) implies that $2 x=-x$; that is to say, $3 x=0$ for any vector $x$, and we conclude that $3=0$ in $\mathbb{K}$.

Now suppose there are two non-zero vectors $x, y \in X$ such that $y \neq \pm x$ and we will arrive to a contradiction.

Condition (SC1) implies that the characteristic of $\mathbb{K}$ is not 2 , and, hence, $x+y \neq x-y$. Without loss of generality we may also assume that

$$
\|y\| \leq\|x\| \quad \text { and } \quad\|x-y\| \leq\|x+y\| .
$$

If $\|y\|<\|x\|$, then $x+y$ and $x-y$ are distinct vectors with the same norm, $\|x+y\|=\|x\|=$ $\|x-y\|$, and whose sum mantains the norm

$$
\|(x+y)+(x-y)\|=\|2 x\|=\|x\|=\|x+y\|,
$$

in contradiction with (SC2).

If $\|y\|=\|x\|$, then (SC2) implies the absurd chains of inequalities

$$
\begin{aligned}
& \|x+y\|<\|x\|=\|2 x\|=\|(x+y)+(x-y)\| \leq \max \{\|x+y\|,\|x-y\|\}, \\
& \|x-y\|<\|x\|=\|2 x\|=\|(x+y)+(x-y)\| \leq \max \{\|x+y\|,\|x-y\|\} .
\end{aligned}
$$

\section{Acknowledgments}

Supported in part by DGICYT projects MTM2016-76958-C2-1-P and PID2019-103961GB-C21 (Spain), ERDF funds and Junta de Extremadura programs GR-15152, IB-16056 and IB-18087.

\section{References}

[1] KubzDela, A. Isometries, Mazur-Ulam theorem and Alexsandrov problem for non-Archimedean normed spaces. Nonlinear Anal. 75, 2012, 2060-2068.https://doi:10.1016/j.na.2011.10.006

[2] Moslehian, M. S. and Sadeghi, G. A Mazur-Ulam theorem in non-Archimedean normed spaces. Nonlinear Anal. 69(10), 2008, 3405-3408.

*Corresponding author: Departamento de Matemáticas, Universidad de Extremadura, Avenida DE Elvas S/N, 06006; BADAJOz. Spain

Email address: coco@unex.es, navarrogarmendia@unex.es 